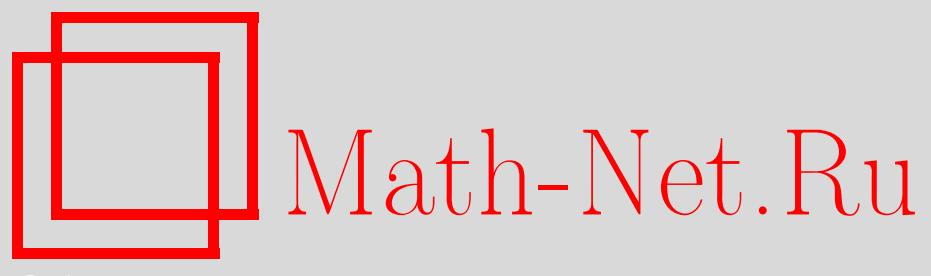

Ш. М. Насибов, О самоканализации решений нелинейного эволюционного уравнения Шрёдингера, Матем. заметки, 2011, том 90, выпуск 5, 789-792

DOI: https://doi.org/10.4213/mzm6351

Использование Общероссийского математического портала Math-Net.Ru подразумевает, что вы прочитали и согласны с пользовательским соглашением http://www . mathnet.ru/rus/agreement

Параметры загрузки:

IP : 54.196 .121 .252

26 апреля 2023 г., 02:24:30

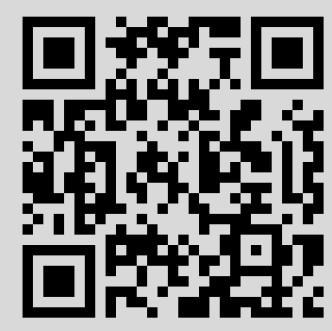




\section{О самоканализации решений нелинейного эволюционного уравнения Шрёдингера}

\section{Ш. М. Насибов}

1. Введение и результаты. Пусть для нелинейного эволюционного уравнения Шрёдингера поставлена задача Коши:

$$
\begin{gathered}
i \frac{\partial u}{\partial \mathrm{t}}=\Delta u+f\left(|u|^{2}\right) u \quad \text { в } \mathbb{R}^{n} \times \mathbb{R}_{+}, \\
\left.u\right|_{t=0}=u_{0}(x) \quad \text { в } \mathbb{R}^{n} .
\end{gathered}
$$

Здесь $f(s)$ - заданная функция на $[0, \infty), u_{0}$ - заданная функция в $\mathbb{R}^{n}$.

Будем принимать стандартные обозначения из монографии Лионса [1]: $H\left(\mathbb{R}^{n}\right)=L^{2}\left(\mathbb{R}^{n}\right)$, $H^{1}\left(\mathbb{R}^{n}\right)=W_{2}^{1}\left(\mathbb{R}^{n}\right)$ - комплексные гильбертовые пространства Соболева; $\|\cdot\|$ - норма в $L_{2}\left(\mathbb{R}^{n}\right),\|\cdot\|_{p}-$ норма в $L_{p}\left(\mathbb{R}^{n}\right), p \geqslant 1$.

ЛЕмма 1 (о ЛОКАЛЬНОм СУЩЕствовАнии). Пусть $u_{0} \in H^{1}\left(\mathbb{R}^{n}\right), f(0)=0, f(s) \in$ $C^{1}([0 ; \infty))$. Тогда найдется такое $t_{0}>0$, что задача $(1),(2)$ имеет единственное локальное решение в классе функций

$$
C^{0}\left(\left[0, t_{0}\right) ; H^{1}\left(\mathbb{R}^{n}\right)\right) \cap C^{1}\left(\left[0 ; t_{0}\right) ; H^{-1}\left(\mathbb{R}^{n}\right)\right)
$$

с альтернативой: или это решение по времени продолжсается на $[0, \infty)$, или это решение продолжается на максимальный интервал существования $\left[0, t_{\max }\right)$, где $t_{\max } \geqslant t_{0}$, причем $\|u(\cdot, t)\|_{H^{1}\left(\mathbb{R}^{n}\right)} \rightarrow+\infty$ npu $t \rightarrow t_{\max }^{-}$.

Лемма 1 доказывается методом сжимающих отображений, после сведения задачи (1), (2) к соответствующему интегральному уравнению (см. [2]).

Для дальнейшего изложения примем следующие обозначения.

Пусть $u(x, t)$ - максимальное решение задачи (1), (2). Положим

$$
\begin{gathered}
A(t)=A(u(t))=\|\nabla u(\cdot, t)\|^{2} \\
E(t)=E(u(t))=A(t)-B(t), \quad \text { где } \quad B(t)=\int_{\mathbb{R}^{n}} F\left(|u(x, t)|^{2}\right) d x,
\end{gathered}
$$

где $F(s)=\int_{0}^{s} f(\tau) d \tau ; a(t)=a(u(t))=\|u(\cdot, t)\|^{2}$,

$$
P_{k}(t)=P_{k}(u(t))=\frac{i}{2} \int_{\mathbb{R}^{n}}\left(u \frac{\partial \bar{u}}{\partial x_{k}}-\bar{u} \frac{\partial u}{\partial x_{k}}\right) d x, \quad k=1,2, \ldots, n .
$$

Лемма 2. Пусть выполнены условия леммы 1. Пусть и $(x, t)$-максимальное решение задача (1), (2) из класса

$$
C^{0}\left(\left[0, t_{\max }\right) ; H^{1}\left(\mathbb{R}^{n}\right)\right) \cap C^{1}\left[\left[0 ; t_{\max }\right) ; H^{-1}\left(\mathbb{R}^{n}\right)\right) .
$$

Тогда задача (1), (2) имеет следующие законы сохранения, т.е. $n p u \forall t \in\left[0, t_{\max }\right)$ справедливы следующие интегральные соотношения:

$$
\begin{aligned}
& a(u(t))=a\left(u_{0}\right), \\
& E(u(t))=E\left(u_{0}\right), \\
& P_{k}(u(t))=P_{k}\left(u_{0}\right), \quad k=1,2, \ldots, n .
\end{aligned}
$$


Доказательства инвариантов (3)-(5) относительно нелинейной шредингеровской динамики (1) несложны, и могут быть найдены в [3].

ОПредЕление 1 . Будем говорить, что глобальное решение $u(x, t)$ задачи $(1),(2)$ из класса

$$
C^{0}\left([0,+\infty) ; H^{1}\left(\mathbb{R}^{n}\right)\right) \cap C^{1}\left[[0 ;+\infty) ; H^{-1}\left(\mathbb{R}^{n}\right)\right)
$$

самоканализируется, если при $\forall t \in[0,+\infty)$ для него выполнена следующая двустороняя оценка: const $\leqslant\|u(\cdot, t)\|_{4} \leqslant$ const, где через const обозначены различные положительные постоянные, не зависящие от $t$ и $u(x, t)$.

Справедливы следующие теоремы.

Теорема 1 (о ГЛОБАЛЬНом СУЩЕСтвовАнии). Пустъ $u_{0} \in H^{1}\left(\mathbb{R}^{n}\right), f(0)=0 f(s) \in$ $C^{2}([0, \infty))$ на интервале $[0,+\infty)$ положительная, возрастающая, выпуклая вниз функиия, m.e. $f(s) \geqslant 0, f^{\prime}(s)>0, f^{\prime \prime}(s)<0 \forall s \in[0,+\infty)$. Тогда при $n=1$ задача (1), (2) имеет глобальное решение из класса

$$
C^{0}\left([0,+\infty) ; H^{1}\left(\mathbb{R}^{n}\right)\right) \cap C^{1}\left[[0 ;+\infty) ; H^{-1}\left(\mathbb{R}^{n}\right)\right) .
$$

При $n=2$ сказанное остается в силе при выполнении условия

$$
\left\|u_{0}\right\|^{2}<\frac{27 \pi}{16 f^{\prime}(0)}
$$

Теорема 2 (о САмоКАНАЛИЗАЦИи). Пусть выполнены все условия теоремы 1. Далее

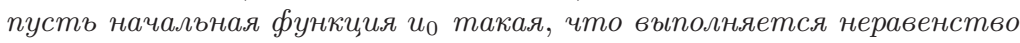

$$
E\left(u_{0}\right)<\frac{P^{2}\left(u_{0}\right)}{a\left(u_{0}\right)},
$$

где $P^{2}\left(u_{0}\right)=\sum_{k=1}^{n} P_{k}^{2}\left(u_{0}\right)$. Тогда решение задачи (1), (2) самоканализируется.

\section{2. Доказательство теорем 1 и 2.}

ДоКАЗАТЕЛЬСТво тЕОРЕмы 1 вытекает из следующего предложения.

УтвеРЖДЕние 1. Пусть выполнены все условия теоремы 1. Тогда $n p u \forall t \in\left[0, t_{\max }\right)$ справедлива следующая априорная оценка:

$$
A(u(t)) \leqslant \text { const. }
$$

Доказательству утверждения 1 предпошлем следующее предложение.

ПреДЛОЖЕНИЕ 1 . Пусть $\rho \in(0,+\infty)$ nрu $n=1,2 ; \rho \in(0,4 /(n-2))$ npu $n \geqslant 3$. Пусть $v(x) \in H^{1}\left(\mathbb{R}^{n}\right)$. Тогда справедливо следующее интерполяционное неравенство ГальярдоНиренберга-Соболева:

$$
\|v\|_{\rho+2} \leqslant k_{0}\|\nabla v\|^{\alpha}\|v\|^{1-\alpha}
$$

где $\alpha=\rho n /[2(\rho+2)], k_{0}$ определяется следующим образом:

$$
k_{0}=k k_{B}\left(\frac{\rho+2}{\rho+1}\right), \quad \text { дде } \quad k=\frac{1}{\sqrt{\alpha^{\alpha}(1-\alpha)^{1-\alpha}}}\left(\frac{\sigma_{n} B}{2}\right)^{\alpha / n}, \quad \sigma_{n}=\frac{2 \pi^{n / 2}}{\Gamma(n / 2)},
$$

$B=B(n / 2, n(1-\alpha) / 2 n)-$ бета-функиия Эйлера;

$$
k_{B}(p)=\left[\frac{(p / 2 \pi)^{1 / p}}{\left(p^{\prime} / 2 \pi\right)^{1 / p^{\prime}}}\right]^{n / 2}, \quad \frac{1}{p}+\frac{1}{p^{\prime}}=1 .
$$


Доказательство этого интегрального неравенства может быть найдено в [4], [5], а также в [6]-[8]. Значение $k_{0}$ приведено в [8].

Для доказательства утверждения 1 воспользуемся неравенством Иенсена [9] и законами сохранения (3), (4). Сначала заметим, что $F(s) \leqslant f(s) s \forall s \in[0,+\infty)$ в силу условия $f^{\prime}(s)>0 \forall s \in[0, \infty)$. Далее справедлива следующая цепочка соотношений:

$$
\begin{aligned}
A(t) & \leqslant\left|E\left(u_{0}\right)\right|+\int_{\mathbb{R}^{n}} F\left(|u|^{2}\right)|u|^{2} d x \leqslant\left|E\left(u_{0}\right)\right|+\int_{\mathbb{R}^{n}} f\left(|u|^{2}\right)|u|^{2} d x \\
& \leqslant\left|E\left(u_{0}\right)\right|+\int|u|^{2} d x f\left(\frac{\int|u|^{4} d x}{\int_{\mathbb{R}^{n}}|u|^{2} d x}\right)
\end{aligned}
$$

(в силу неравенства Иенсена)

$$
\begin{aligned}
& =\left|E\left(u_{0}\right)\right|+\int\left|u_{0}\right|^{2} d x f\left(\frac{\int|u|^{4} d x}{\int\left|u_{0}\right|^{2} d x}\right) \\
& =\left|E\left(u_{0}\right)\right|+\int\left|u_{0}\right|^{2} d x f\left(\frac{k_{0}^{4}\left\|u_{0}\right\|^{4-n} A^{n / 2}(t)}{\left\|u_{0}\right\|^{2}}\right)
\end{aligned}
$$

(в силу неравенства $(8)$ при $n \leqslant 3$ ), т.е. получаем следующее неравенство:

$$
A(t) \leqslant\left|E\left(u_{0}\right)\right|+\left\|u_{0}\right\|^{2} f\left(k_{0}^{4}\left\|u_{0}\right\|^{2-n} A^{n / 2}(t)\right) .
$$

По формуле Маклорена для $f(s)$ имеем

$$
f(s)=f(0)+f^{\prime}(0) s+\frac{f^{\prime \prime}(\theta s)}{2} s^{2},
$$

где $0<\theta<1$. Отсюда в силу условий на $f(s)$ получаем $f(s)<f^{\prime}(0) s$. Благодаря этой оценке из $\left(9_{1}\right)$ имеем

$$
A(t) \leqslant\left|E\left(u_{0}\right)\right|+f^{\prime}(0) k_{0}^{4}\left\|u_{0}\right\|^{2-n} A^{n / 2}(t) .
$$

При $n=1$ из $\left(9_{2}\right)$ вытекает априорная оценка (7).

Пусть $n=2$. Тогда при $\rho=2$ выполнено $k_{0}=2 / \sqrt[4]{27 \pi}$. Если $f^{\prime}(0) k_{0}^{4}\left\|u_{0}\right\|^{2}<1$, то из $\left(9_{2}\right)$ следует априорная оценка (7). Доказательство теоремы 1 завершено.

Доказательство теоремы 2. Легко убедиться в том, что справедливо следующее интегральное соотношение:

$$
A\left(e^{i q x} u\right)=A(u)-2 q P(u)+q^{2} a(u),
$$

где $q x=\sum_{k=1}^{n} q_{k} x_{k}, q P(u)=\sum_{k=1}^{n} q_{k} P_{k}, q^{2}=\sum_{k=1}^{n} q_{k}^{2}$. В соотношении (10), полагая $q_{k}=P_{k} / a$, получаем

$$
A\left(e^{i q x} u\right)=A(u)-\frac{P^{2}(u)}{a(u)},
$$

где $P^{2}(u)=\sum_{k=1}^{n} P_{k}^{2}(u)$.

Из (11), используя законы сохранения (3)-(5), имеем

$$
A\left(e^{i q x} u\right)-B(u)=E\left(u_{0}\right)-\frac{P^{2}\left(u_{0}\right)}{a\left(u_{0}\right)},
$$

откуда в силу условия

получаем

$$
E\left(u_{0}\right)<\frac{P^{2}\left(u_{0}\right)}{a\left(u_{0}\right)}
$$

$$
\frac{P^{2}\left(u_{0}\right)}{a\left(u_{0}\right)}-E\left(u_{0}\right)<B(u)
$$


Ho

$$
B(u)<a\left(u_{0}\right) f\left(\frac{k_{0}^{4}}{\left\|u_{0}\right\|^{2}}\|u(\cdot, t)\|^{4}\right),
$$

отсюда и из (12) заключаем, что

$$
\frac{\left\|u_{0}\right\|^{2}}{k_{0}^{4}} f^{-1}\left(\frac{P^{2}\left(u_{0}\right)}{a^{2}\left(u_{0}\right)}-\frac{E\left(u_{0}\right)}{a\left(u_{0}\right)}\right) \leqslant\|u(\cdot, t)\|^{4} .
$$

Далее из оценки $A(u(t)) \leqslant$ const в силу неравенства (8) и закона сохранения (3) имеем

$$
\|u(\cdot, t)\|_{4} \leqslant \text { const. }
$$

Из (13) и (14) следует, что

$$
c_{1} \leqslant\|u(\cdot, t)\|_{4} \leqslant c_{2},
$$

где $c_{1}$ и $c_{2}$ - некоторые положительные постоянные, не зависящие от $t, u(x, t)$, а зависящие от $u_{0}$.

Теорема 2 доказана (см. также [10]).

\section{СПИСОК ЦИТИРОВАННОЙ ЛИТЕРАТУРЫ}

[1] Ж.-Л. Лионс, Некоторые методы решения нелинейных краевых задач, Мир, М., 1972. [2] J. Ginibre, G. Velo, J. Funct. Anal., 32:1 (1979), 1-32. [3] Ш. М. Насибов, Дифферени. уравнения, 16:4 (1980), 660-670. [4] E. Gagliardo, Ricerche Mat., 7 (1958), 102-137. [5] L. Nirenberg, Ann. Scuola Norm. Sup. Pisa (3), 13 (1959), 115-162. [6] H. A. Levine, Ann. Mat. Pura Appl. (4), 124 (1980), 181-197. [7] E. J. M. Veling, JIPAM. J. Inequal. Pure Appl. Math., 3:4 (2002), Article 63. [8] Ш. М. Насибов, Докл. АН СССР, 307:3 (1989), 538-542. [9] Г. Г. Харди, Дж. Е. Литтльвуд, Г. Полиа, Неравенства, ИЛ, М., 1948. [10] Ш. М. Насибов, Докл. АН СССР, 285:4 (1985), 807-811.

Ш. М. Насибов

Институт прикладной математики Бакинского государственного университета E-mail: nasibov_sharif@hotmail.com
Поступило

21.10.2008

Исправленный вариант

17.06.2010 